\title{
THE PATHOLOGY OF HANDWRITING AS A RESULT OF DRUG ABUSE. A CASE STUDY
}

\author{
V. G. Băncilă
}

\section{Violeta-Gabriela Băncilă}

Police Academy "Alexandru Ioan Cuza", Bucharest, Romania

*Correspondence: Violeta-Gabriela Băncilă, Police Academy "Alexandru Ioan Cuza", 1-3 Aleea Privighetorilor St., District 1, 014031 Bucharest, Romania E-Mail: gabriela_bnc@yahoo.com

\begin{abstract}
The case study highlights the importance of observing the damaged handwriting which occurs in drug abuse. Handwriting of a drug consumer presents certain characteristics which are useful to be known and analyzed by certain specialists (forensic experts, psychiatrists) involved in research in both Criminal and Medical field. Handwriting analysis can become a valuable medical diagnosis tool for monitoring the progress made by drug addicts receiving medication; the conclusions reveal the characteristics of damaged handwriting due to drug abuse, after analyzing several signatures of a 24 year old subject.
\end{abstract}

Keywords: pathology, drugs, handwriting, signature, marijuana.

\section{Introduction}

Handwriting, this "fine seismograph", tends to be sensitive to external agents. Drugs and hallucinogens of all kinds, as well as alcohol, leave their mark on the neuromuscular system.

Although their effects may vary, there are certainties about a couple of issues. Factors which can create changes in the individual's handwriting are: the handwriting's degree of development, changes caused by writing with the "hand driven ", alcohol, poisoning, drug abuse, drugs, toxic substances, stress; alteration of handwriting as a result of physical agents such as age, fatigue, decrease or loss of vision, amputation of fingers or hand $^{l}$.

Heroin, cocaine (and their varieties) addicts, same as alcoholics, feel better initially after taking the drug, as the neuromuscular acuity and their performance is improved; The opposite state is abstinence or withdrawal. Studies have shown that under the influence of drugs like LSD -25 and BOL - 148 the handwriting and signature suffer changes similar to those altered due to alcohol consumption.

Frequent cases occur on handwritten wills or document signatures, shortly before or after the ingestion of an overdose that eventually killed the victim. Checks and receipts are sometimes signed under the influence of drugs, but after recovering to normal, the person it was no longer to remember all that they have signed.

\section{The Use of handwriting analysis studies on drug behavior}

Studying the handwriting of drug users is useful for highlighting both health state after ingesting hallucinogenic substances and also the patient's progress over the treatment

\footnotetext{
${ }^{1}$ Nicoleta-Elena Buzatu, "Drugs Consumption - a Handwriting Modifier", International Journal of Criminal Investigation vol. 2, Issue 4/2012, p. 274.
} 
administrated by the doctor. We know that toxic ingestion, whether it is inhaled or administrated intravenously induces changes in the central nervous system (CNS ), affecting the writing mode - the physiological mechanism of writing is the result of temporary nerve connections and conditioned reflexes, drugs acting as an external factor which corrupts the way letters are made.

Altered writing will depend primarily on age and also other factors (internal and / or external) that may change the individual's handwriting. Internal factors refer to the preexisting neuropsychiatric diseases - such as Multiple Sclerosis (MS), paralysis of a limb, major depression, schizophrenia, etc. Also, if mixing alcohol with drugs or antidepressant medication, a sample of such writing will provide an increased degree of damage. A similar situation can occur if studying samples of letters from an elderly person, be it only occasionally consuming hallucinogenic substances. In such case, the deterioration factor (drug) overlaps the other factors previously occurred and would enrich the writing's damage degree.

If we refer to the consumers' age, it should be noted that the degree of alteration of the handwriting is proportional to its stability. We know that writing is stabilized around the age of 18-20 years along with achieving maturity of the individual, the writing becomes automatic and movements are simplified.

Also important is the frequency, on which an individual writes - in recent years, due to the increasing role of computers in everyday life, there are fewer and fewer jobs where employees are forced to carry documents in hand. Under such circumstances, we conclude that future graphic expertise and handwriting analysis will be performed on samples of already having a preexistent deterioration. Nowadays, the use of drugs has increased significantly among Romanian young people.

It should be noticed that the process of detoxification is not always successful; studying the patient's handwriting during the detoxification treatment and after its completion will gradually reveal improvements in letters' execution in proportion to the progress made by the individual.

Regarding the external factors, we refer to the conditions in which the handwritten test was conducted: the existence of a proper scriptural instrument, appropriate support, other optimal conditions ( staying seated, absence of disturbances such as loud noises, etc. ).

In order to complete the assessment of the effects of drugs on the graphic sample it would be needed for each investigated person and each kind of drug substance, to analyze handwriting into four distinct phases: 1 . before drug use, 2. during narcotic administration 3 . in withdrawal stage 4 in the process of detoxification. Such research should be extended to a large number of subjects, in order to reach conclusive results, to be statistically significant for to be able to use them in future practices.

Regarding the deterioration of the handwriting, there are always cases arguing about the degree of impairment of drug - affected writing or on the time of detoxification or withdrawal phases. Problems arising in the field of handwriting analysis are huge for the following reasons:

a) because of the diversity of the phases in which writing samples were obtained - it's almost impossible to have samples of subjects in the same psychophysical condition; b ) for the comparative samples dating from the period prior to administration of the drug, technical obstacles may exist, and the procedure, in particular where the script refers to a long period of time - there may also be other internal unknown factors to alter the sample. c ) qualitative differences emerging from fulfilling comparison with the result that it could be mistaken for signs of forgery ( considering it wrong to be a fake by imitation of the handwriting ) or would be difficult to explain without a careful analysis of the sample of handwriting in question that the deterioration is actually the consequence of substance abuse. 


\section{General characteristics of writing psychopathology}

The deterioration of any handwriting will depend on the dose and type of the administered drug (-taking into consideration also the internal and external factors mentioned before).

After conducting an experiment by Hirsch, Jarvik and Abramson ${ }^{2}$ which required doses of LSD, BOL, alcohol, ergometrine, methamphetamine hydrochloride and scopolamine given to subjects, it was observed that under the influence of LSD, alcohol and BOL 's, $60 \%$ of subjects increased their handwriting, while for the remaining subjects the effect was exactly the opposite.

The largest number of errors, cuts, corrections and erasures was highlighted after scopolamine's administration, while after administration of LSD the following issues like: the largest irregularly reflected on font size, also word spacing, slope, and lack of control in the execution of letters occurred.

Another study made by Legge, Steinberg and Summerfield ${ }^{3}$ on the effects of different strength $\mathrm{NOH}$ doses administration to patients, substance which is known as strong acting anesthetic central nervous system, relevant results have shown such as increasing the baseline length (horizontal dimension words); this could explain why $\mathrm{NOH}$ causes a systematic increase in the size of writing that is correlated with the concentration of administered doses, but it suggests that it acts on the neuromuscular system to such an extent that it can achieve rude movements, so the effects being different from the ones caused by the alcohol.

Following laboratory studies which demonstrated that caffeine consumption improves psychomotor performances, writing and signature become smoother and the speed of execution faster, the same effects were obtained after administration of methamphetamine. ${ }^{4}$

High consumption of marijuana ${ }^{5}$ (less than 5 cigarettes) has the effect of increasing the size of the letters, paragraphs deviations and careless execution of proper letters, so moderate consumption of marijuana has less visible effects on writing than alcohol.

Variability of subjective reactions to psychotropic stimuli substances is explained with such skill by Hans Gross: "it seems that psychological stability affects the extent and direction of writing which will change after drug ingestion. We have reason to believe that graphical reactions occurred after drug administrations are proportional to the individual's psychological reactions".

\section{Case study on the signature of a Drug consumer}

The case study was performed over a 24 years old male (S.A.) - occasional consumer of marijuana which had executed several signatures after ingested the specified amount of drug.

A total number of 8 signatures have been analyzed; we can observe the deterioration of the subject's signature in a total time of 4 days. For better observation the grid method was used, applying a transparent grid ruler over every signature (size of a square $5 \mathrm{~mm}$ ).

\footnotetext{
${ }^{2}$ Hirsch, M. W., Jarvik, M. E., and Abramson, H. A., Lysergic Acid Diethylamide (LSD-25): XVIII Effects of LSD-25 and Six Related Drugs upon Handwriting. Journal of Psychology, 1956; 41: pp 11-22).

${ }^{3}$ Legge, David, Steinberg, Hannah, and Summerfield, Arthur, Simple Measures of Handwriting as Indices of Drug Effects. Perceptual and Motor Skills, 1964; 18: pp. 549-558.

${ }^{4}$ Dhawan, B. N., Bapat, S. K., and Saxena, V. C., Effect of Four Centrally Acting Drugs on Handwriting. Japanese Journal of Pharmacology, 1969; 19: pp. 63-67.

5 Foley, Bobby G., and Miller, A. Lamar, The Effects of Marijuana and Alcohol Usage on Handwriting. Presented at the meeting of the International Association of Forensic Sciences (Wichita, KS, 1978).
} 


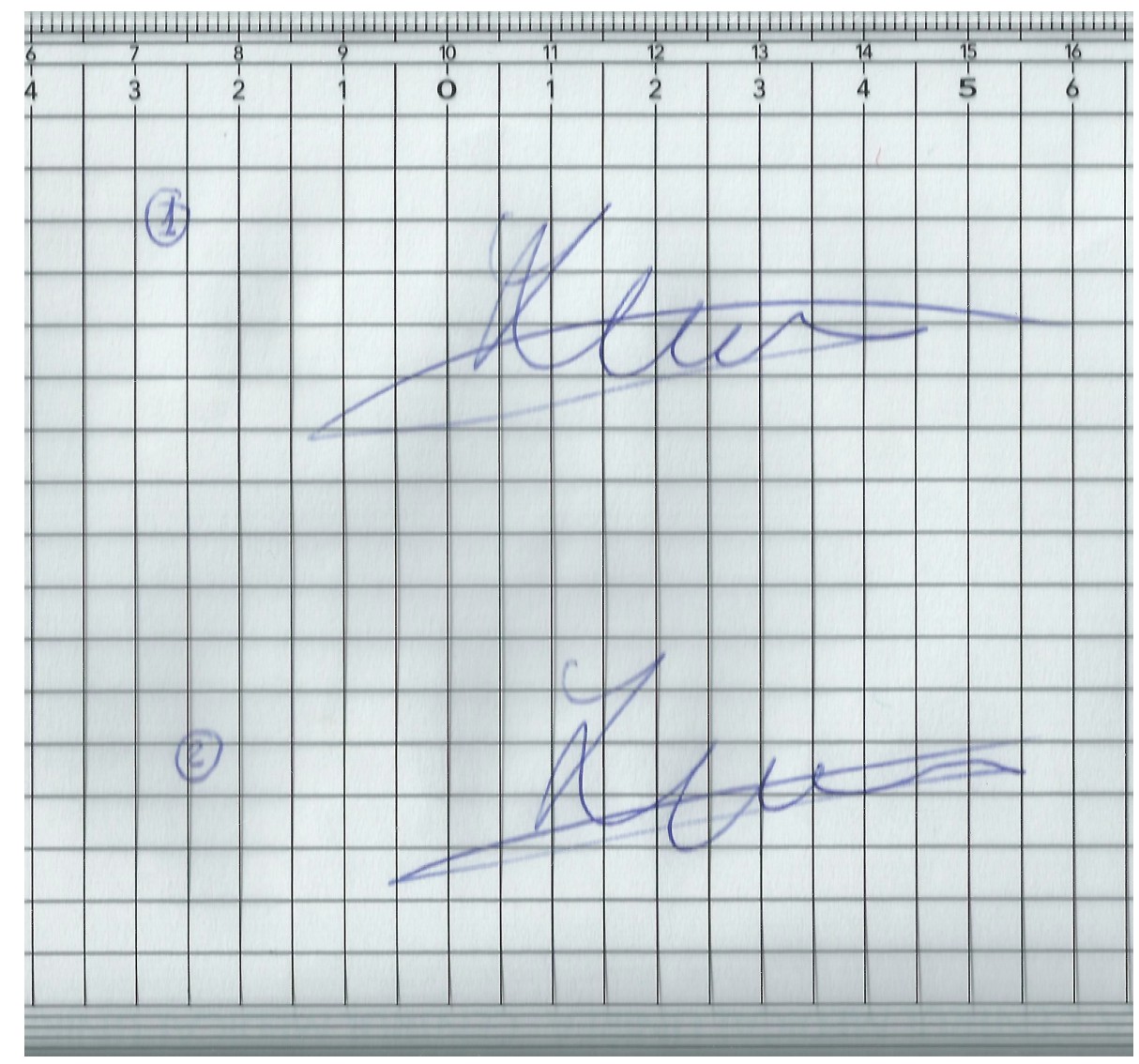

Day 1: Signature made approximately 12 hours after consuming a dose of $0.3 \mathrm{~g}$ marijuana;

Day 2: Signature made 7 hours after consuming the same dose;

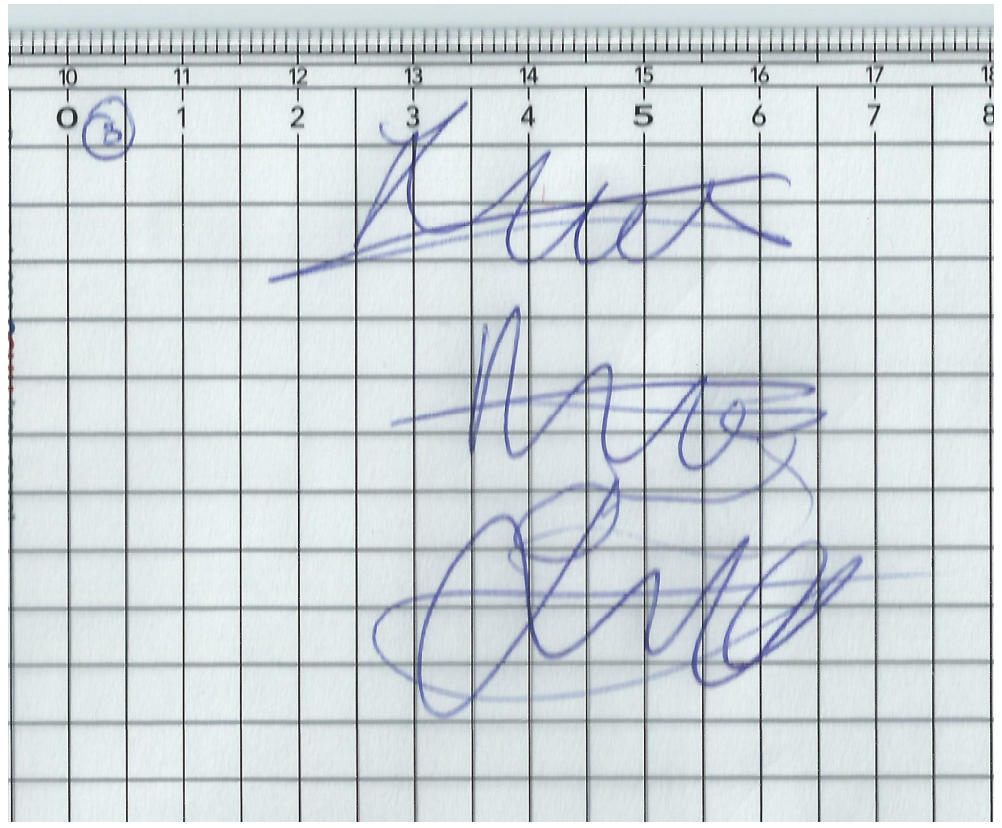

Day 3: Signature made after consuming the same dose; the first signature largely retains its general characteristics, and we observe a slight decrease on the horizontal. When trying to 
repeat the signature, the subject fails to properly execute it, his attempt is visible in the next two tries (low speed, lack of coordination, visible tremor). At a superficial forensic examination, the expert may wrongfully consider the $2^{\text {nd }}$ and $3^{\text {rd }}$ attempts as imitations of the first signature.

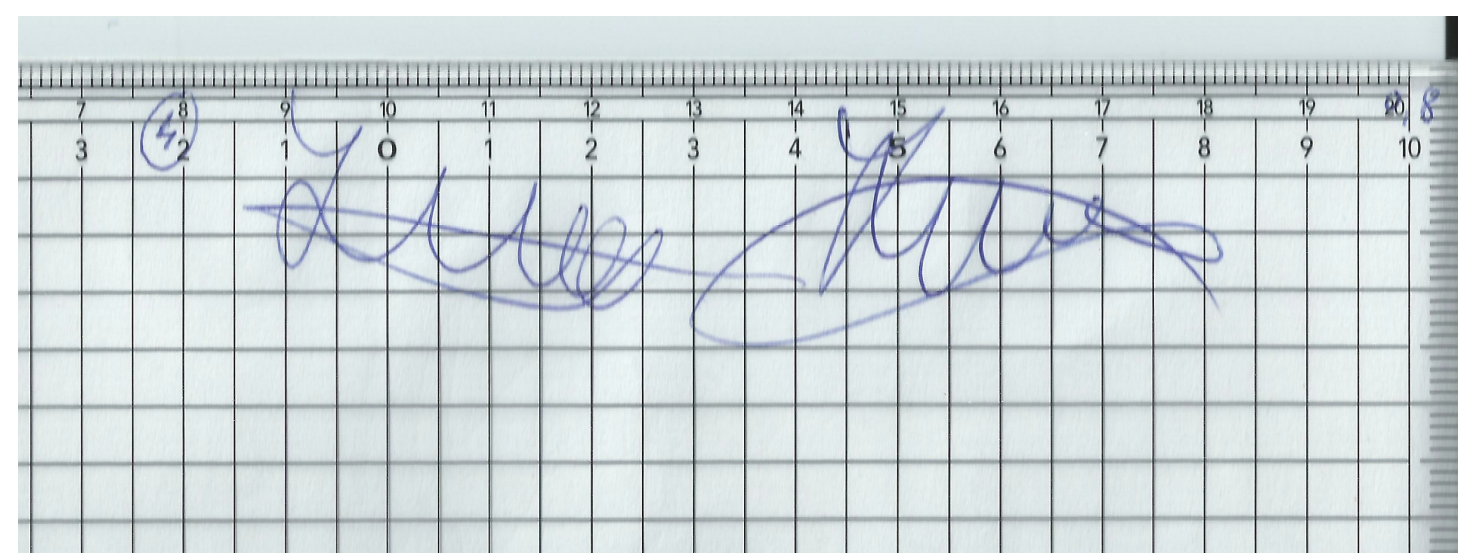

Day 4: Maintaining the same time schedule as before, but after increasing the dose approximately 3 times than the usual (consuming a total of $0.8 \mathrm{~g}$ of marijuana) we observe the signature is performed with great difficulty, requiring two attempts to succeed. The first signature is characterized by downward baseline; by the end of the signature we observe visible failures of execution and a totally low rate of successful achievement.

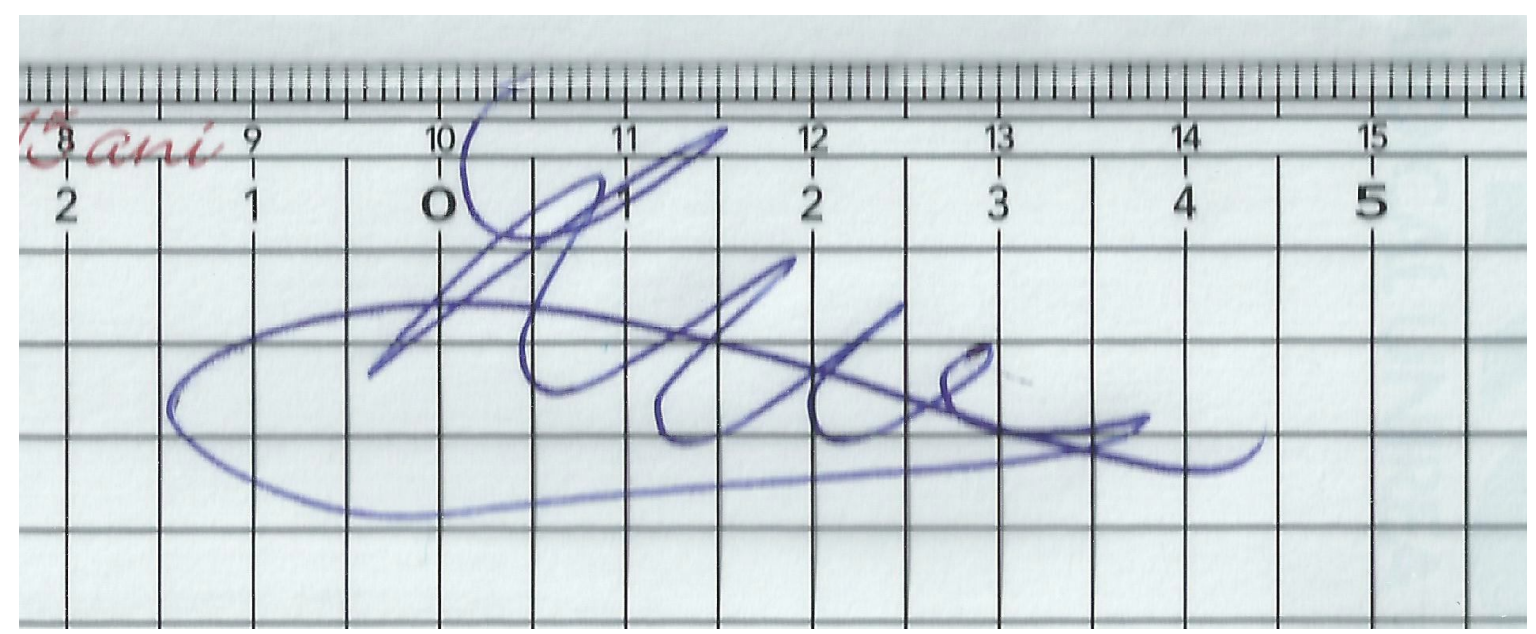

Signature made by the subject after a period of 2 weeks of abstinence: the baseline maintains a downward baseline and the execution pressure remains high. The execution speed is improved, as well as the coordination movements.

\section{Conclusions}

In conclusion, the effects of drugs on the graphic gesture shapes can be briefly classified as: - inconstant pressure; - inconsistency and incoherence in keeping slant left/ right; - performing individual letters with different slants, - sloppiness and mistakes ( based on impaired endocrine or muscle spasticity problems ); - sudden deviations, irregular amplitudes and vertically - horizontally superficiality and disorder in the general organization of writing in page; - differences in pressure between the upper and the lower zones due to exhaustion and relaxation of muscle tone; - tendency to transform the angular shapes into threaded movements. 
Note that general characteristics should always be analyzed after taking into consideration the subject's medical history (previous mental illness or any other diseases that can alter his general health status), the age, the type of drug used (narcotics or even pharmacy medicines).

\section{Bibliography}

Nicoleta-Elena Buzatu, "Drugs Consumption - a Handwriting Modifier "International Journal of Criminal Investigation”, vol. 2, Issue 4/2012;

Karen Kristin Amend, Mary Stansbury Ruiz, Handwriting Analysis, New Page Books 1980 Franklin Lakes N.J.;

Foley, Bobby G., and Miller, A. Lamar, The Effects of Marijuana and Alcohol Usage on Handwriting. Presented at the meeting of the International Association of Forensic Sciences (Wichita, KS, 1978);

Hirsch, M.W., Jarvik, M.E., and Abramson, H.A., Lysergic acid diethylamide (LSD -25): XVIII Effects of LSD -25 and Six Related Drugs upon Handwriting. Journal of Psychology, 1956, 41: pp. 11-22;

Legge, David Steinberg, Hannah, and Summerfield, Arthur, Simple Measures of Handwriting as Indices of Drug Effects, Perceptual and Motor Skills, 1964;

Dhawan, BN, Bapat, SK, and Saxena, VC, Effect of Four Centrally Acting Drugs on Handwriting, Japanese Journal of Pharmacology, 1969. 